\title{
Viés Atencional no Abuso de Drogas: Teoria e Método ${ }^{1}$
}

\author{
Ana Carolina Peuker ${ }^{2}$ \\ Fernanda Machado Lopes \\ Lisiane Bizarro \\ Universidade Federal do Rio Grande do Sul
}

\begin{abstract}
RESUMO - O viés atencional pode eliciar fissura, diminuir a concentração em tarefas não relacionadas à droga e aumentar a vulnerabilidade à recaída em dependentes de drogas. O objetivo deste estudo é discutir visões teóricas recentes e principais métodos de investigação do papel do viés atencional nos comportamentos aditivos. Realizou-se busca nas bases de dados Medline, Pubmed e Lilacs. Essa busca revelou que a dot-probe task e o teste emocional de Stroop estão entre os principais métodos de investigação do viés atencional. Também foram apontadas limitações metodológicas nas investigações sobre viés atencional, sugerindo que esse fenômeno deve ser estudado sob condições melhor controladas, que considerem níveis de dependência, privação e fissura. Estudar o viés atencional pode contribuir para identificar mecanismos cognitivos subjacentes aos comportamentos aditivos.
\end{abstract}

Palavras-chave: viés atencional; abuso de drogas; teste emocional de Stroop; dot-probe task.

\section{Attentional Bias in Drug Abuse: Theory and Method}

\begin{abstract}
Attentional bias to drug-related cues can induce craving, decrease concentration on non-related drug tasks and increase vulnerability to relapse in drug addicts. The aim of this study is to discuss current theories and research methods about the attentional bias role on addictive behaviors. The literature review of Medline, Pubmed and Lilacs databases showed that the dot-probe task and the emotional Stroop test are among the main methods of attentional bias investigation. This review also pointed out the methodological limitations in attentional bias research, suggesting that this phenomenon should be studied under better controlled conditions, which should consider levels of dependence, withdrawal and craving. Further studies on attentional bias can help to understand cognitive processes that underlie addictive behaviors.
\end{abstract}

Keywords: attentional bias; drug abuse; emotional stroop task; dot-probe task.

A atenção prioriza seleção e monitoramento de determinados estímulos sobre outros e, como tal, media cognição, emoção e comportamento tanto para selecioná-los, como para evitá-los (Schoenmakers, Wiers, Jones, Bruce \& Jansen, 2007). O viés atencional pode ser definido como uma prontidão particular para processar certos estímulos mais do que outros, devido ao valor apetitivo atribuído a eles (Williams, Mathews \& MacLeod, 1996). Modelos cognitivos de psicopatologia consideram que o viés atencional desempenha um papel central na etiologia e na manutenção dos distúrbios emocionais (Williams \& cols., 1996).

Indivíduos afetados por transtornos emocionais como ansiedade, depressão, fobias, transtornos de stress pós-traumático e transtornos alimentares demonstram um aumento da atenção em direção aos eventos relacionados à patologia que apresentam (Dobson \& Dozois, 2004; Williams \& cols., 1996). Da mesma forma, na dependência química, o viés atencional para pistas associadas ao uso de drogas é refe-

1 Apoio Financeiro: Coordenação de Aperfeiçoamento de Pessoal de Nível Superior (Capes), sob forma de bolsa de Doutorado (primeira autora).

2 Endereço para correspondência: Universidade Federal do Rio Grande do Sul, Instituto de Psicologia, Laboratório de Psicologia Experimental, Neurociências e Comportamento (LPNeC). Rua Ramiro Barcelos 2600, sala 106. Porto Alegre, RS. CEP 90035-003. E-mail: acpeuker@terra.com.br. rido como tendo um importante papel na transição do uso ocasional à dependência, no desejo subjetivo persistente de consumir a droga (fissura) e na recaída (Lopes, Peuker \& Bizarro, 2009).

No caso dos comportamentos aditivos, postula-se que as pistas ambientais, associadas ao reforço produzido pelo uso da droga, adquirem saliência. Assim, as pistas condicionadas aos efeitos da droga recebem mais atenção do usuário em detrimento de outros estímulos do meio. De forma geral, usuários tendem a direcionar sua atenção preferencialmente para estímulos do ambiente associados à sua droga de escolha (Bradley, Mogg, Wright \& Field, 2003; Ehrman \& cols., 2002; Lubman, Peters, Mogg, Bradley \& Deakin, 2000; Robbins \& Ehrman, 2004; Robinson \& Berridge, 1993, 2003; Schoenmakers \& cols., 2007; Tiffany, 1990; Williams \& cols., 1996).

Sugere-se uma importante classificação dos tipos de estímulos e seus possíveis efeitos. O estímulo apetitivo, reforçador ou recompensador é aquele que provoca sensação de prazer ou alívio de algo desagradável (e.g., comida, dinheiro). O estímulo aversivo ou punitivo é o que provoca desconforto físico ou psicológico (e.g., frio, insulto). Por último, o estímulo neutro é aquele considerado insignificante, que por si só não causaria sensação de prazer nem desconforto (Catania, 1992/1999). No caso dos adictos, a droga é considerada um estímulo apetitivo à medida que causa sensação de prazer ou de alívio do desconforto da fissura. 
Este estudo foi desenvolvido com o objetivo de apresentar e discutir abordagens teóricas atuais e os principais métodos de investigação do papel do viés atencional nos comportamentos aditivos. $\mathrm{O}$ método consistiu em realizar uma busca nas bases de dados Medline, Pubmed e Lilacs, com as palavras-chave: viés atencional (attentional bias), pistas relacionadas à droga (drug cues) e abuso de substâncias (substance abuse). Os artigos selecionados obedeceram a critérios de inclusão que consideraram pertinência, instrumentos de pesquisa utilizados (DotProbe Task, também denominada Visual-Probe Task, e o Teste Emocional de Stroop) e sugestões para futuras investigações. A fundamentação teórica não contemplou pesquisas nacionais porque as metodologias utilizadas para investigação do viés atencional eram discrepantes com as do objetivo deste estudo.

\section{Viés Atencional e Comportamentos Aditivos: Modelos Teóricos}

É consenso entre os estudiosos do comportamento aditivo que o estímulo associado à droga induz respostas condicionadas que podem motivar seu consumo. Logo, a motivação para consumir substâncias de abuso tais como álcool, nicotina, opiáceos e cocaína é determinada, em grande parte, pelas propriedades motivacionais das drogas e das pistas ambientais associadas a elas. O estímulo relacionado ao uso da droga produz uma variedade de respostas associadas aos seus efeitos, incluindo fissura, excitação e abstinência. O viés atencional parece estar correlacionado a maior frequência e intensidade dessas respostas (reatividade a pistas), pois mantém os indivíduos focalizados no uso da droga. Por essa razão, a tendência para direcionar e manter a atenção para esses estímulos, ou viés atencional, pode desempenhar um papel decisivo no uso de drogas e na recaída (O’Brien, Childress, Ehrman \& Robbins, 1998; Robinson \& Berridge, 1993, 2003; Tiffany, 1990).

Algumas teorias sustentam que respostas (incluindo disposição para beber, sentimentos agradáveis) que seguem a exposição às pistas associadas às drogas devem-se ao condicionamento clássico. Modelos cognitivos do uso de drogas também reconhecem a importância desses eventos ambientais. Por exemplo, o modelo de expectativa de Marlatt (1985) e o do processamento automático de Tiffany (1990) apoiam a ideia de que eventos ambientais servem como gatilhos para o uso de drogas. Nesse contexto, a reatividade às pistas associadas à droga pode representar um importante fator de risco para transição do uso ocasional à dependência e também para a recaída (Robbins \& Ehrman, 2004). Modelos baseados na abstinência (withdrawal) consideram que pistas relacionadas à droga podem eliciar estados negativos, semelhantes à abstinência (withdrawallike) (Siegel, 1979). Assim, o uso de droga seria motivado pela busca de alívio desses estados desagradáveis (drugopposite). Por outro lado, teorias do incentivo sustentam que as pistas relacionadas à droga eliciam estados semelhantes à droga (drug-like) e, assim, motivariam a ingestão por relembrar o usuário das propriedades reforçadoras do uso (Stewart, deWit \& Eikelboom, 1984).
Embora atribuam diferentes padrões de respostas às pistas ambientais, esses dois modelos compartilham duas importantes características. Ambos sustentam que eventos ambientais associados com o uso de drogas tornam-se estímulos condicionados. Os dois modelos propõem que as respostas condicionadas eliciadas por pistas relacionadas à droga podem favorecer o uso repetido da droga, levando à dependência e à recaída.

A teoria do incentivo-sensitização, de Robinson e Berridge (1993), postula que as drogas alteram os sistemas cerebrais relacionados à recompensa que servem de mediadores da saliência do incentivo. Consequentemente, esses circuitos neurais tornam-se hipersensíveis, ou sensibilizados, para os efeitos específicos da droga e estímulos associados a ela. Um estímulo inicialmente neutro torna-se um incentivo saliente, atraente e desejado, destacando-se dentre muitos estímulos do ambiente aos quais o usuário de drogas poderia dirigir a atenção. Esse fenômeno foi chamado de saliência do incentivo. A capacidade de focalizar a atenção em atividades não relacionadas à droga fica prejudicada, favorecendo a transição do uso ocasional à dependência ou à recaída quando na abstinência da droga. A teoria do incentivo-sensitização propõe que, embora as relações entre drogas e pistas sejam aprendidas, o que contribui de fato para a transição do uso ocasional para a dependência de drogas é o impacto motivacional dessas pistas, ou seja, sua habilidade para mobilizar sistemas cerebrais relacionados à motivação (Robinson \& Berridge, 1993, 2003).

Uma visão teórica alternativa sobre o comportamento aditivo, proposta por Tiffany (1990), é a teoria do processamento automático, que também enfatiza a capacidade do estímulo relacionado à droga chamar atenção do usuário em detrimento de outros estímulos do meio. Nesse modelo, derivado da teoria do processamento da informação, o autor propõe que o comportamento de busca da droga é determinado por um processo automático. Com o uso repetido da droga, os estímulos relacionados a ela (e.g., imagens, sons, odores) passam a integrar uma rede associativa armazenada na memória. Quando o usuário é exposto a esses estímulos, esquemas de ação são ativados, desencadeando um forte desejo e a necessidade de consumir a droga (Johnsen, Laberg, Cox, Vaksdal \& Hugdahl, 1994; Lubman \& cols., 2000; Tiffany, 1990; Townshed \& Duka, 2001).

Tanto a teoria do incentivo-sensitização de Robinson e Berridge (1993, 2003) quanto o modelo do processamento cognitivo automático proposto por Tiffany (1990) destacam a capacidade do estímulo relacionado à droga de chamar mais a atenção do usuário do que outros estímulos do meio. Essas duas teorias sustentam a ideia de que o viés atencional desempenha um importante papel no desenvolvimento, manutenção e vulnerabilidade à recaída dos comportamentos aditivos (Johnsen \& cols., 1994; Lubman \& cols., 2000; Robinson \& Berridge, 1993, 2003; Tiffany, 1990; Townshed \& Duka, 2001). Mais recentemente, tem sido sugerido que o incentivo e o hábito podem desempenhar diferentes papéis na aquisição e manutenção da dependência de drogas, dependendo da severidade do uso de substâncias (Di Chiara, 2000).

Durante os estágios iniciais do aprendizado sobre o uso da droga e das pistas ambientais, fatores de aprendizagem relacionados ao incentivo controlam primariamente a resposta 
operante. Depois de uma resposta operante ter sido aprendida e desempenhada repetidamente, o comportamento é emitido automaticamente após o estímulo antecedente (Mogg, Field \& Bradley, 2005). Por exemplo, Di Chiara (2000) considera que o comportamento de fumar, nos estágios iniciais da dependência, é controlado essencialmente por um processo de aprendizado relacionado ao incentivo. Como resultado da liberação de dopamina, pistas associadas ao fumar adquirem propriedades motivacionais. Com o tempo, o comportamento de fumar passa a ser determinado por respostas baseadas no hábito aprendido. Portanto, em fumantes com maiores níveis de dependência, o comportamento de fumar é provavelmente um hábito que é iniciado automaticamente, eliciado pelo estímulo associado à droga, sem a presença de um processo de motivação-incentivo (Everitt, Dickinson \& Robbins 2001).

Estudiosos do comportamento de abuso de substâncias estão interessados no viés atencional porque ele está relacionado com o processo da aprendizagem e da habituação do uso da droga (Robinson \& Berridge, 1993, 2003; Tiffany, 1990; Townshed \& Duka, 2001). Na dependência e na recaída, muitos adictos referem um comportamento automatizado, sem intenção, no qual evitar o consumo frente ao estímulo provocador torna-se extremamente difícil (Tiffany, 1990). Sendo assim, o estudo do viés atencional pode contribuir para o aperfeiçoamento de habilidades de enfrentamento orientadas para abstinência (Monti, Kadden, Rohsenow, Cooney \& Abrams, 2002/2005; Robbins \& Ehrman, 2004).

Em relação ao processo atencional, cabe salientar que distinções têm sido feitas entre os mecanismos envolvidos na orientação inicial da atenção e na manutenção da atenção. Acredita-se que subsistemas neurais separados estejam envolvidos nessas diferentes operações cognitivas. A orientação inicial é um processo relativamente rápido, mais automatizado, que pode ser avaliado quando a duração da exposição dos estímulos apresentados for curta (100-200 ms). Já a manutenção da atenção é provavelmente mais influenciada por variáveis motivacionais e pode ser avaliada quando os estímulos são apresentados com uma duração maior (2000 ms) (Field, Mogg, Zatteler \& Bradley, 2004).

\section{Viés Atencional: Principais Métodos de Investigação}

Entre os principais paradigmas utilizados para investigar o viés atencional estão o teste de Stroop e a dot-probe task (Ehrman \& cols., 2002). Na versão original do teste de Stroop (Stroop, 1935) são apresentadas séries de palavras escritas em diferentes cores, sendo os participantes instruídos a ignorar o conteúdo semântico das mesmas e a se concentrar apenas na nomeação da cor em que elas estão impressas. Esse método tem demonstrado que os participantes, de forma típica, apresentam tempos de reação maiores ao nomearem palavras que estão incongruentes com a cor em que estão impressas (e.g., palavra "verde" impressa com tinta amarela) do que as que estão congruentes (e.g., palavra "verde" impressa com tinta verde).

O paradigma de Stroop tem sido utilizado para estudar o viés atencional em diversas populações clínicas, demonstrando que os participantes tendem a nomear mais lentamente a cor das palavras relacionadas aos seus problemas, estímulos emocionalmente relevantes, do que aquelas com valência emocional neutra. Esse fenômeno é conhecido como efeito emocional de Stroop (Cox, Brown \& Rowlands, 2003; Williams \& cols., 1996). Nessa tarefa, o viés atencional é mensurado a partir dos tempos de leitura das palavras emocionalmente relevantes para o indivíduo. Há evidências de que indivíduos com transtorno de humor, como depressão, ou com transtornos ansiosos, como fobias e transtorno de estresse pós-traumático, nomeiam mais lentamente a cor das palavras relacionadas aos seus problemas. Isto é, tendem a direcionar sua atenção para estímulos relacionados à sua patologia (Williams \& cols., 1996). O paradigma emocional de Stroop também tem sido utilizado para investigar os mecanismos cognitivos responsáveis pelo desenvolvimento e manutenção de comportamentos aditivos (Bauer \& Cox, 1998; Cox \& cols., 2003; Cox, Yeates \& Regan, 1999; Johnsen \& cols.,1994; Sharma, Albery \& Cook, 2001; Stormark, Laberg, Nordby \& Hugdahl, 2000; Waters \& Green, 2003).

Por meio do paradigma de Stroop, demonstrou-se que indivíduos que abusavam ou dependiam de álcool apresentavam tempos de leitura maiores quando expostos a estímulos relacionados ao álcool (Bauer \& Cox, 1998; Johnsen \& cols., 1994; Sharma \& cols, 2001; Stormark \& cols., 2000). Além dos dependentes e abusadores, demonstrou-se que bebedores ocasionais também apresentaram viés atencional para estímulos relacionados ao álcool (Bruce \& Jones, 2004; Lusher, Chandler \& Ball, 2004). Um estudo realizado com bebedores frequentes de álcool revelou que, no teste emocional de Stroop, eles apresentaram tempos de leitura maiores para palavras relacionadas ao álcool do que para palavras neutras (Cox \& cols., 1999). Em outro estudo, também com o teste emocional de Stroop, constatou-se que palavras relacionadas ao álcool interferiram no processo atencional tanto de alcoolistas e bebedores-problema quanto de bebedores sociais (Bauer \& Cox, 1998; Sharma \& cols., 2001). Portanto, há evidência de que bebedores em geral possuem viés na atenção para estímulos relacionados ao álcool quando verificados pelo paradigma emocional de Stroop. Sugere-se que a magnitude desses efeitos pode variar diretamente com o nível do consumo habitual de álcool (Cox \& cols., 1999).

Esses resultados demonstraram também a importância do viés atencional na compreensão do abuso e dependência de drogas e podem ser entendidos de duas formas: em termos da teoria do incentivo-sensitização (Robinson e Berridge, 1993, 2003), na qual o estímulo associado à droga de escolha captura a atenção do usuário; ou segundo a teoria do processamento automático (Tiffany, 1990), que considera que o estímulo relacionado à droga ativa os esquemas de ação que levam ao comportamento automático de uso.

Outro método empregado para investigar o viés atencional consiste em uma tarefa de atenção visual (dot-probe task). Nessa tarefa, um par de estímulos (figuras ou palavras) é apresentado de forma simultânea, na tela de um computador. Em seguida, os dois estímulos desaparecem e um deles é imediatamente substituído por uma seta. Os participantes são orientados a indicar o mais rapidamente possível a direção para a qual a seta aponta (para cima ou para baixo). A latência das respostas serve como um indicador de atenção visual para o estímulo apresentado (Townshend \& Duka, 
2001). Ou seja, tempos de reação menores quando a seta substitui uma classe particular de estímulos indica viés na atenção para essa classe. Esse paradigma foi primeiramente empregado para avaliar o viés atencional em populações clínicas. Observou-se que indivíduos ansiosos, quando comparados com controles normais, apresentavam viés atencional para estímulos emocionalmente ameaçadores (MacLeod, Mathews \& Tata, 1986). Em geral, observa-se que o nível de viés atencional está diretamente associado ao estado emocional atual do indivíduo. De acordo com isso, um estudo com pacientes ansiosos revelou que, após terem realizado o tratamento com sucesso, indivíduos não demonstraram mais viés atencional em relação ao grupo controle (Mogg, Mathews \& Eysenck, 1992).

Atualmente, a dot-probe task tem sido amplamente utilizada em investigações sobre os comportamentos aditivos (Robbins \& Ehrman, 2004). O viés atencional para estímulos relacionados à droga já foi observado, por meio dessa tarefa, em dependentes de opiáceos (Lubman \& cols., 2000), em fumantes (Ehrman \& cols., 2002) e em bebedores frequentes (Field \& cols., 2004; Townshend \& Duka, 2001). De forma geral, usuários de drogas apresentam tempos de reação menores quando a seta substitui estímulos (palavras ou imagens) relacionados à sua droga de escolha, do que quando a seta reaparece no lugar de estímulos neutros. Participantes dependentes de opiáceos, mantidos com metadona, demonstraram tempos de reação menores na dot-probe task quando o alvo (seta) era substituído por imagens relacionadas à droga, sugerindo um viés na atenção para esse tipo de estímulo (Lubman \& cols., 2000).

O movimento dos olhos de fumantes foi examinado enquanto eles executavam a dot-probe task. Os participantes mantiveram a fixação do olhar em direção às pistas associadas ao fumar por períodos mais longos do que para pistas controle. Além disso, constatou-se que o viés atencional estava associado à fissura (Mogg \& cols., 2003) e que o viés atencional poderia ser potencializado por estados de privação de nicotina (Field, Mogg \& Bradley, 2004b).

Observou-se, ainda, por meio desse mesmo paradigma, que usuários frequentes de álcool apresentavam maior viés na atenção para imagens relacionadas ao álcool quando comparados a bebedores ocasionais de álcool (Townshend \& Duka, 2001). Ou seja, a atenção dos usuários de álcool estava focalizada, essencialmente, para as pistas relacionadas à droga. Em conjunto, essas evidências corroboram tanto a teoria do incentivo-sensitização de Robinson e Berridge (1993) quanto o modelo teórico do processamento cognitivo automático proposto por Tiffany (1990). Esses dois modelos teóricos salientam a capacidade do estímulo relacionado à droga se destacar e chamar a atenção do usuário em detrimento de outros estímulos do meio.

\section{Desafios Metodológicos e Direções Futuras para Estudos do Viés Atencional nos Comportamentos Aditivos}

Diversos estudos têm empregado os paradigmas emocional de Stroop e a dot-probe task para investigar o viés atencional em usuários de drogas. Em geral, estudos utilizando a dot-probe task têm produzido resultados positivos mais consistentes que o paradigma emocional de Stroop. Contudo, nenhum desses paradigmas tem evidenciado um conjunto de dados homogêneo (Bauer \& Cox, 1998; Field, Mogg \& Bradley, 2004a; Field \& cols., 2004b; Robbins \& Ehrman, 2004; Sharma \& cols., 2001; Townshend \& Duka, 2001).

Muitos pesquisadores que investigam o viés atencional nos comportamentos aditivos tendem a ignorar importantes distinções entre a orientação inicial e a manutenção da atenção (Field \& cols., 2004). A maior parte dos resultados da literatura que utiliza a dot-probe task tem sido obtida com apresentações de estímulos rápidos (500 ms ou menos), que refletem reações relativas à orientação inicial da atenção. Entretanto, alguns estudos têm demonstrado efeitos usando durações maiores de estímulos, de $1000 \mathrm{~ms}$ ou mais, que requerem a manutenção da atenção voluntária para o estímulo emocionalmente relevante (Robbins \& Ehrman, 2004).

O tipo de mudança atencional capturada pela dotprobe task possui dois componentes: a mudança inicial na atenção para o estímulo de interesse e, posteriormente, a manutenção da atenção. Estudos com fumantes revelaram que o viés atencional pode operar durante todo o processo cognitivo da atenção (Ehrman \& cols., 2002; Field \& cols., 2004). Contudo, bebedores frequentes, em comparação com bebedores ocasionais, apresentaram viés atencional para imagens relacionadas ao álcool expostas por tempos maiores (2000ms), mas não quando as imagens relacionadas ao álcool foram expostas em tempo curto $(200 \mathrm{~ms})$. Isso sugere que o viés na orientação visual para pistas relacionadas ao álcool em bebedores frequentes de álcool opera principalmente na manutenção da atenção (Field \& cols., 2004).

Conforme demonstrado, tanto o paradigma emocional de Stroop como a dot-probe task podem ser utilizados para mensurar o viés atencional. Contudo, o resultado obtido em cada uma dessas tarefas deve ser interpretado de forma distinta. O efeito Stroop está baseado na ideia que palavras relacionadas a transtornos emocionais causam interferência no processamento da informação. Dessa forma, os indivíduos nomeariam a cor dos estímulos emocionalmente relevantes mais lentamente do que os estímulos com valência emocional neutra, refletindo em tempos de reação maiores. Já o viés atencional na dot-probe task resultaria da alocação da atenção para o estímulo de interesse, facilitando o desempenho na tarefa. Assim, o viés atencional seria expresso por tempos de reação menores para os estímulos emocionalmente relevantes.

Alguns problemas metodológicos tornam a interpretação dos resultados da tarefa emocional de Stroop ambígua. Essa tarefa pode ser apresentada de duas formas: no formato consecutivo e alternado. No primeiro, os estímulos pertencentes a uma determinada categoria (e.g., palavras relacionadas à ansiedade) são todos apresentados sequencialmente em um mesmo bloco. Já no formato alternado, os estímulos neutros e emocionais são apresentados um a um, de forma intercalada (Robbins \& Ehrman, 2004).

No formato de consecutivo, em decorrência da forma de apresentação em bloco, o desempenho na tarefa pode ser afetado por efeitos cumulativos (carryover). Isso pode ocorrer em função da magnitude da interferência das palavras emocionalmente relevantes que se seguem uma após a outra, 
consecutivamente. Nessa modalidade de apresentação, cada classe de estímulos, emocional ou neutro, é apresentada em conjunto na tela de um computador ou em cartões separados. Assim, o efeito cumulativo pode aumentar a interferência emocional no teste de Stroop. Inversamente, no formato não-bloco, os estímulos-alvo ou palavras emocionalmente relevantes e os estímulos controle são apresentados de forma intercalada. Nesse caso, a interferência na nomeação de cores produzida pelas palavras emocionalmente relevantes pode repercutir na resposta à próxima palavra da lista, aumentando o tempo de leitura do estímulo neutro (Waters, Sayette \& Wertz, 2003).

Palavras controle apresentadas em blocos isolados não sofrem influência de efeitos carryover. Contudo, palavras controle apresentadas na modalidade não bloco, intercaladas com estímulos-alvo, podem ser influenciadas justamente por precederem estímulos emocionalmente relevantes. Consequentemente, os efeitos cumulativos decorrentes do formato intercalado podem reduzir a magnitude do efeito Stroop. Nos dois casos, os efeitos cumulativos parecem ser inconsistentes com uma interpretação atencional do efeito Stroop que atribui a interferência na nomeação de cores aos efeitos da palavra apresentada naquele momento (Robbins \& Ehrman, 2004).

A maior interferência na nomeação de cores de palavras emocionalmente relevantes comparadas com palavras controle é geralmente tida como evidência de que tais estímulos comandam a atenção seletiva. Entretanto, outras explicações para resultados oriundos do teste emocional de Stroop são possíveis (Williams \& cols., 1996). Palavras relacionadas à preocupação atual do indivíduo podem ativar memórias (efeito prime) que podem interferir na nomeação das cores. Palavras emocionalmente relevantes também poderiam produzir estados emocionais (e.g., ansiedade), causando interferência na nomeação da cores, não apenas em virtude do viés atencional para certa classe de estímulos, mas também por uma ruptura da concentração ou de uma desorganização geral do comportamento causado pelo impacto emocional. Além disso, as palavras relacionadas ao transtorno emocional de um indivíduo poderiam causar interferência na nomeação de cores mais como resultado de sua familiaridade do que pela sua habilidade de comandar a atenção seletiva (Robbins \& Ehrman, 2004).

Muitas das dificuldades metodológicas encontradas na tarefa de Stroop podem ser evitadas na dot-probe task. Essa tarefa oferece evidências mais diretas sobre a orientação da atenção para eventos relacionados ao estímulo emocional. Além disso, ela está menos sujeita aos efeitos carryover já descritos. Isso porque, nessa tarefa, os estímulos emocionais e neutros são apresentados simultaneamente e a localização dos estímulos também é variada a cada tentativa. Assim, eles podem aparecer tanto do lado esquerdo quanto do lado direito do monitor do computador, na mesma proporção. Portanto, a atenção dirigida para o lado esquerdo ou direito do monitor em uma tentativa favorecerá da mesma forma tanto o estímulo controle quanto o emocional na próxima tentativa (Robbins \& Ehrman, 2004).

No teste de Stroop, os participantes atendem a um estímulo de cada vez enquanto que na dot-probe task, um par de estímulos (imagens ou palavras) é apresentado simultanea- mente, tornando possível avaliar mudanças na atenção entre os dois estímulos, independentemente do nível educacional e habilidades de leitura. Dessa forma, a dot-probe task representa melhor as condições com as quais os indivíduos se defrontam em seu ambiente natural. Por exemplo, no caso de indivíduos que abusam de drogas, tanto estímulos relacionados como aqueles não relacionados à droga competem pela atenção visual do usuário no meio no qual está inserido (Ehrman \& cols., 2002). Ou seja, por examinar mudanças na atenção visual entre estímulos emocionais e controle, a dot-probe task modela situações do ambiente natural no qual o indivíduo é confrontado com múltiplos eventos que competem por sua atenção (Robbins \& Ehrman, 2004). Também se deve levar em consideração que um estímulo pictório pode ser mais efetivo ou mais ecologicamente válido para investigar o viés atencional do que um estímulo textual (Bruce \& Jones, 2004; Lubman \& cols., 2000).

$\mathrm{O}$ estudo do viés atencional para pistas relacionadas à droga oferece a possibilidade de elucidar os mecanismos cognitivos envolvidos na fissura, na transição do uso ocasional para a dependência e na recaída (Lopes \& cols., 2008; Robinson \& Berridge, 1993, 2003; Tiffany, 1990; Townshed \& Duka, 2001). A atenção para eventos relacionados às drogas pode gerar a expectativa do futuro reforço propiciado por ela e, com isso, motivar comportamentos de busca. O viés atencional para pistas associadas à droga pode utilizar a capacidade limitada de mecanismos cognitivos necessários para habilidades de coping orientado para abstinência. Na abordagem da terapia de Beck para abuso de substâncias, os pacientes são instruídos a adotar novas atitudes em relação ao uso de drogas. O viés atencional pode distrair indivíduos do uso de tais estratégias cognitivas, especialmente em situações de risco (Monti \& cols., 2002/2005; Robbins \& Ehrman, 2004).

O estudo da relação entre viés atencional nos comportamento aditivos também pode trazer outros benefícios. Medidas de viés atencional podem ser úteis para avaliar o nível de motivação para a abstinência do uso de drogas, ou seja, a prontidão para tentar abstinência ou mesmo a probabilidade de recaída após o tratamento (Sayette, Martin, Wertz, Shiffman \& Perrott, 2001; Lopes \& cols., 2008). Resultados alternativos poderão ser produzidos se medidas de viés atencional e comportamento de uso de drogas se correlacionarem, pois as medidas biológicas não são facilmente obtidas e as escalas tradicionais de autorelato nem sempre fornecem dados confiáveis, uma vez que as respostas podem ser distorcidas pelos usuários de droga (Robbins \& Ehrman, 2004).

O desempenho de usuários de drogas na tarefa de Stroop e na dot-probe task tem sido interpretado como uma demonstração do viés atencional para o estímulo relacionado à droga. Entretanto, muitas investigações não incluem grupos controle ou estímulos de comparação, tornando difícil concluir que os resultados refletem uma resposta atencional específica para eventos pessoalmente relevantes relacionados à droga. Esses estudos iniciais, embora promissores, precisam prosseguir com demonstrações do viés atencional em condições melhor controladas e com investigações relacionando os níveis de viés com outras medidas de consumo de drogas (Robbins \& Ehrman, 2004). 
Há evidência de que o viés atencional para estímulos relacionados à droga associa-se com desejo subjetivo intenso e persistente fissura de consumir a substância. Embora na maioria dos estudos sobre o viés atencional no abuso de substâncias não tenham sido consideradas medidas de fissura dos participantes e não tenham sido feitas correlações entre viés atencional e fissura, sugere-se que há uma associação robusta entre essas variáveis. Tal relação já foi demonstrada entre abusadores de álcool (Field \& cols., 2004) e usuários de maconha (Field \& cols., 2004a).

Por exemplo, Field e cols.(2004a) não encontraram diferenças quanto ao viés em usuários de maconha comparados com controles não usuários. Contudo, quando os dados foram reavaliados dividindo os usuários em dois grupos, com dois níveis de fissura (alto e baixo), encontrou-se uma interação entre grupo e posição da seta. Usuários de maconha que reportaram maiores níveis de fissura direcionavam a atenção para pistas associadas à droga, ao contrário de usuários com baixa fissura e abstêmios.

Deve-se salientar que esses diferentes estudos variaram amplamente em relação à amostra investigada (usuários de múltiplas substâncias de abuso; usuários ativos versus aqueles que buscaram tratamento), aos instrumentos de medida do viés atencional (tarefa de Stroop ou dot-probe task) e às medidas de fissura empregadas (escalas análogas visuais de item único versus questionários multi-item e multifatoriais). Algumas pesquisas não evidenciaram correlação significativa entre viés atencional e fissura (Ehrman \& cols., 2002; Lubman \& cols., 2000). Em estudos nos quais diferentes medidas de viés atencional foram utilizadas, a fissura não se correlacionou com todas as medidas, apenas com algumas (Mogg \& Bradley, 2002; Mogg \& cols., 2003). Até o presente, as evidências disponíveis não permitem conclusões precisas sobre as circunstâncias sob as quais há uma forte associação entre essas duas variáveis. Por isso, a investigação sistemática desse tópico deve ser tida como um importante objetivo para novos estudos (Field \& Cox, 2008).

A magnitude do viés parece sofrer influência da privação da droga. Modelos da adição (Robinson \& Berridge, 1993, 2003; Tiffany, 1990) postulam que há um aumento da atenção para pistas associadas à droga sob condições de privação. Robinson \& Berridge $(1993,2003)$ teorizaram que pistas associadas à droga podem ganhar saliência em função do aumento da fissura, que pode ser induzida por meio da privação. Tiffany (1990) propõe que há um aumento do viés para estímulos associados à droga quando tentativas de uso da droga são evitadas. Sendo assim, futuramente, o viés atencional poderia ser investigado considerando o nível de fissura dos participantes. É possível que fossem encontradas diferenças entre grupos com alta e baixa fissura, como observado por Field e cols. (2004b). Além disso, outras manipulações que potencializem a fissura podem ser testadas a fim verificar seu impacto sobre o viés atencional. Além da privação, exposição a pistas e priming, a indução de estados de humor negativos também pode manipulada (Field \& Cox, 2008).

Resultados provenientes de estudos recentes sugerem que a relação causal entre fissura e viés atencional é recíproca. Isto é, o viés atencional parece aumentar a fissura. Ou seja, quando as pistas relacionadas à droga tornam-se o foco da atenção, elas podem eliciar a fissura. Da mesma maneira, quando a fissura está aumentada o estímulo associado à droga torna-se mais saliente, destacando-se e chamando mais a atenção do usuário (viés atencional aumentado). $\mathrm{O}$ conhecimento acerca dos efeitos recíprocos produzidos pelo viés atencional e pela fissura pode subsidiar o desenvolvimento de intervenções clínicas mais efetivas. Nesse sentido, intervenções que auxiliem os usuários deslocar seu foco atencional das pistas associadas à droga e, assim, minimizar o viés atencional para esses estímulos, podem contribuir para melhores prognósticos.

Técnicas de validade e confiabilidade precisam ser empregadas na avaliação do viés atencional. Se os escores do viés atencional podem ser considerados fortes indicadores do abuso de substâncias, medidas de teste e re-teste necessitam ser estabelecidas. Até o momento, não existem estudos longitudinais utilizando o teste de Stroop nem a dot-probe task com usuários de drogas.

Também tem sido sugerido que o viés atencional pode servir como uma medida de resultado em estudos de eficácia de tratamento. Se níveis de viés atencional são controlados pela gravidade do transtorno emocional, então o esbatimento da sintomatologia durante o tratamento deveria ser acompanhado pela redução no nível de viés atencional (Mogg \& cols., 1992; Robbins \& Ehrman, 2004). Novas investigações poderiam demonstrar quais níveis de viés atencional estão diretamente implicados na gravidade dos comportamentos aditivos. Para isso, as medidas de viés atencional precisariam ser correlacionadas a outros indicadores de severidade clínica ou resultado. Assim, medidas de viés poderiam servir como preditores de prognóstico ou gravidade do transtorno. Também seria válido investigar, em estudos posteriores, outras variáveis pertinentes, como tempo de privação, frequência do uso e abstinência da droga, nas medidas de processamento cognitivo. Dessa forma, o estudo do viés atencional poderia contribuir para identificar os mecanismos cognitivos subjacentes aos comportamentos aditivos.

\section{Referências}

Bauer, D., \& Cox, W.M. (1998). Alcohol-related words are distracting to both alcohol abusers and non-abusers in Stroop colornaming task. Addiction, 93, 1539-1542.

Bradley, B. P., Moog, K., Wright, T., \& Field, M. (2003). Attentional bias in drug dependence: Vigilance for cigarette-related cues in smokers. Psychology of Addictive Behaviors, 17, 66-72.

Bruce, G., \& Jones, B. T. (2004). A pictorial Stroop paradigm reveals an alcohol attentional bias in heavier compared to lighter social drinkers. Journal of Psychopharmacology, 18, 527-33.

Catania, A. C. (1999). Aprendizagem: comportamento, linguagem e cognição (D. D. G. de Souza, Trad.). Porto Alegre: Artes Médicas. (Trabalho original publicado em 1992)

Cox, W. M., Brown, M. A., \& Rowlands, L. J. (2003). The effects of alcohol cue exposure on non-dependent drinker's attentional bias for alcohol-related stimuli. Alcohol \& Alcoholism, 38, 45-49.

Cox, W. M., Yeates, N. G., \& Regan, M. C. (1999). Effects of alcohol cues on cognitive processing in heavy e light drinkers. Drug and Alcohol Dependence, 55, 85-89. 
Di Chiara, G. (2000). Role of dopamine in the behavioral actions of nicotine related to addiction. European Journal of Pharmacology, 392, 295-314.

Dobson, K .S., \& Dozois, D. J. (2004). Attentional biases in eating disorders: A meta-analytic review of Stroop performance. Clinical Psychology Review, 23, 1001-1022.

Ehrman, R. N., Robbins, S. J., Bromwell, M. A., Lankford, M. E., Monterosso, J. R., \& O'Brien, C. P. (2002). Comparing attentional bias to smoking cues in current smokers, former smokers, and non-smokers using a dot-probe task. Drug and Alcohol Dependence, 67, 185-191.

Everitt, B. J., Dickinson, A., \& Robbins, T. W. (2001). The neuropychological basis of addictive behavior. Brains Research Review, 36, 129-138.

Field, M., \& Cox, W. M. (2008). Attentional bias in addictive behaviors: A review of its development, causes, and consequences. Drug and Alcohol Dependence, 97, 1-20.

Field, M., Mogg, K., \& Bradley, B. P. (2004a). Cognitive bias and drug craving in recreational cannabis users. Drug Alcohol Dependence, 74, 105-11.

Field, M., Mogg, K., \& Bradley, B. P. (2004b). Eye movements to smoking cues: Effects of nicotine deprivation. Psychopharmacology, 173, 116-123.

Field, M., Mogg, K., Zatteler, J., \& Bradley, B. (2004). Attentional biases for alcohol cues in heavy and light social drinkers: The roles of initial orienting and maintained attention. Psychoparmacology, 176, 88-93.

Johnsen, H. B., Laberg, C. J., Cox, M. W., Vaksdal, A., \& Hugdahl, K. (1994). Alcoholic subjects' attentional bias in the processing of alcohol-related words. Psychology of Addictive Behaviors, 8, 111-115.

Lopes, F. M., Peuker, A.C., \& Bizarro, L. (2008). Viés atencional em fumantes. Psico (PUCRS), 39, 382-390.

Lubman, D. I., Peters, L. A., Mogg, K., Bradley, B. P., \& Deakin, J. F. W. (2000). Attentional bias for drug cues in opiate dependence. Psychological Medicine, 30, 169-175.

Lusher, J., Chandler, C., \& Ball, D. (2004). Alcohol dependence and the alcohol Stroop paradigm: Evidence and issues. Drug and Alcohol Dependence, 75, 225-231.

Marlatt, G. A. (1985). Cognitive factors in relapse process. Em G. A. Marlatt \& J. R. Gordon (Orgs.), Relapse prevention (pp. 128-200). New York: Guilford.

McLeod, C., Mathews, A., \& Tata, P. (1986). Attentional bias in emotional disorders. Journal of Abnormal Psychology, 95, 15-20.

Mineka, S., \& Sutton, S. K. (1992). Cognitive biases and the emotional disorders. Psychological Science, 3, 65-69.

Mogg, K., Bradley, B. P., Field, M., \& De Houwer, J. (2003). Eye movements to smoking related pictures in smokers: Relationship between attentional biases and implicit and explicit measures of stimulus valence. Addiction, 98, 825-836.

Mogg, K. \& Bradley, B. P. (1992). Selective processing of smoking-related cues in smokers: Manipulation of deprivation level and comparison of three measures of processing bias. Journal of Psychopharmacology, 16, 385-392.

Mogg, K., Field, M., \& Bradley, B. P. (2005). Attentional and approach biases for smoking cues in smokers: An investigation of competing views of addiction. Psychopharmacology, 180, 333-341.

Mogg, K., Mathews, A., \& Eysenck, M. (1992). Attentional bias to threat in clinical anxiety states. Cognition and Emotion, $6,149-159$.
Monti, P. M., Kadden, R. M., Rohsenow, D. J, Cooney, N. L., \& Abrams, D. B. (2005). Tratando a dependência de álcool. Um guia de treinamento das habilidades de enfrentamento (M. G. Armando, Trad.). São Paulo: Roca. (Trabalho original publicado em 2002)

O'Brien, C. P., Childress, A. R., Ehrman, R., \& Robbins, S. J. (1998). Conditioning factors in drug abuse: Can they explain compulsion? Journal of Psychopharmacology, 12, 15-22.

Robbins, S., \& Ehrman, R. (2004). The role of attentional bias in substance abuse. Behavioral and Cognitive Neuroscience Reviews, 3, 243-260.

Robinson, T., \& Berridge, K. (1993). The neural basis of drug craving: An incentive-sensitization theory of addiction. Brain Research Reviews, 18, 247-291.

Robinson, T., \& Berridge, K. (2003). Addiction. Annual Reviews Psychology, 54, 25-53.

Sayette, M. A., Martin, C. S., Wertz, J. M., Shiffman, S., \& Perrot, M. A. (2001). A multi-dimensional analysis of cue-elicited craving in heavy smokers and tobacco chippers. Addiction, 96, 1419-1432.

Schoenmakers, T., Wiers, R., Jones, B., Bruce, G., \& Jansen, A. (2007). Attentional re-training decreases attentional bias in heavy drinkers without generalization. Addiction, 102, 399-405.

Sharma, D., Albery, I. P., \& Cook, C. (2001). Selective attentional bias to alcohol related stimuli in problem drinkers and non-problem drinkers. Addiction, 96, 85-295.

Siegel, S. (1979). The role of conditioning in drug tolerance and addiction. Em J. D. Keehn (Ed.), Psychopathology in animals: Treatment and research implications (pp. 143-168). New York: Academic Press.

Stewart, J., deWit, H., \& Eikelboom, R. (1984). The role of unconditioned and conditioned drug effects in the self-administration of opiates and stimulants. Psychological Review, 91, 251-268.

Stormark, J. M., Laberg, J. C., Nordby, H., \& Hugdahl, K. (2000). Alcoholics`selective attention to alcohol stimuli: Automated processing? Journal of Studies on Alcohol, 61, 18-23.

Stroop, J. R. (1935). Studies of interference in serial verbal reactions. Journal of Experimental Psychology, 18, 643-662.

Tiffany, S. (1990). A cognitive model of drug urges and druguse behavior: Role of automatic and non-automatic processes. Psychological Review, 97, 147-168.

Townshed, J. M., \& Duka, T. (2001). Attentional bias with alcohol cues: Differences between heavy and occasional social drinkers. Psychopharmacology, 157, 67-74.

Waters, A. J., Sayette, M. A., \& Wertz, J. M. (2003). Carryover effects can modulate emotional Stroop effects. Cognition and Emotion, 17, 501-509.

Waters, H., \& Green, M. (2003). A demonstration of attentional bias, using a novel dual task paradigm, towards clinically salient material in recovering alcohol abuse patients? Psychological Medicine, 33, 491-498.

Williams, J. M., Mathews, A., \& MacLeod, C. (1996). The emotional Stroop task and psychopathology. Psychological Buletinl, 120, 3-24.

Recebido em 22.04.08 Primeira decisão editorial em 02.09.08 Versão final em 17.09.08 Aceito em 20.11.08 